\title{
Quantum Effects in the Plasmonic Response
}

\section{JAVIER AIZPURUA}

Center for Materials Physics in San Sebastian (CSIC-UPV/EHU), Spain

\section{ANDREI G. BORISOV}

L'Institut des Sciences Moléculaires d'Orsay, CNRS-Université Paris Sud, France

Excitation of localized surface plasmon resonances in metal nanoparticles and nanoparticle assemblies allows to manipulate light at nanometer scales well beyond the wavelength and opens the possibility of many applications. The complexity of the variety of methods to fabricate plasmonic structures and the sophistication of optical characterization techniques invites for completing the plasmonics landscape with a comprehensive theoretical understanding of the optical response. Classical approaches based on the solution of Maxwell's equations using local (model or empirical) bulk dielectric constant are extensively used to describe plasmonic systems and gain the necessary intuition. At the same time, recent experimental and theoretical developments have clearly demonstrated that as soon as nanometer and subnanometer scales are approached, quantum effects such as tunneling, non-local screening and the atomistic structure of the metal nanoparticles become important. In this chapter, we discuss the major manifestations of quantum effects in the properties of localized plasmons, with particular emphasis on the underlying physics. 


\subsection{Introduction}

Interaction of light with metallic nanoparticles can be greatly enhanced if the incident electromagnetic wave is at resonance with collective electron excitations, so called plasmons. Known for a long time in surface science, where intense research on plasmons has been developed in the context of the interpretation of electron energy loss spectroscopy (EELS) experiments,${ }^{1}$ and studied in detail in the context of cluster physics, ${ }^{2}$ nowadays plasmonic properties of nanostructured materials are receiving renewed attention as a key building block in nanophotonics. ${ }^{3-5}$ Driven by technological progress, plasmonics is now a flourishing field of fundamental and applied research as it allows "to manipulate light at the nanoscale". ${ }^{3}$ Indeed, advances in nanofabrication ${ }^{6,7}$ allow to engineer the plasmonic response of systems and fully exploit strongly enhanced near fields and scattering resonances in far fields produced by plasmonic nanoparticles. ${ }^{3-5,8-12}$ While noble metals such as gold and silver are the most common materials in infrared plasmonics, recent reports address the possibility of using $\mathrm{Al}$ nanoparticles with the perspective to exploit their plasmon resonances in the ultraviolet. ${ }^{13}$

The design of the optimal nanostructure with a specific optical response in the near or far fields requires accurate theoretical predictions. Thus, the availability of theoretical tools capable of modelling, predicting, describing, and interpreting experimental data at formal and numerical levels is of paramount importance. Most of the formal approaches and numerical techniques developed to obtain the optical response of a nanoobject root back to the classical macroscopic Maxwell's equations. ${ }^{5,7,14}$ The non-dispersive local dielectric permittivity is routinely used to describe the optical response of metallic nanoparticles delimited by sharp boundaries. It is only recently that technology has allowed to engineer plasmonic nanostructures where quantum aspects of the motion of conduction electrons significantly affect the optical response. ${ }^{15-19}$ Basically, observing the quantum regime of response implies that the characteristic size(s) of the system is at the nm scale, comparable to the Fermi electron wavelength in the metal. Thus, several studies have been performed on quantum 
effects in the energy and lifetime of the collective plasmon excitations in small metal clusters, ${ }^{2,20-23}$ as well as on photon-assisted electron tunneling through narrow gaps in semiconductor and metal nanostructures in the $\mathrm{THz}$ range. ${ }^{24,25}$ Current research on quantum effects in plasmonics at optical frequencies is mainly focused on two aspects:

(1) Non-locality of the screening, where the spatial profile of the induced electron density cannot be infinitely sharp, as assumed in classical theories.

(2) The coupling between the electromagnetic field and electron tunneling across narrow junctions between metal nanoparticles, where the conductive contact can be established prior to the direct geometrical overlap.

The above-mentioned quantum effects are not captured within standard classical approaches, and so the description of the corresponding plasmonic structures requires new developments. The non-local screening can be modelled within a classical electromagnetic theory framework within the non-local hydrodynamical model ${ }^{26}$ (NLHD) allowing to partially lift such non-physical results as e.g. the divergence of near fields upon narrowing the gap between plasmonic nanoparticles. This theory has recently been greatly improved to include the quantum spill out of the electron density outside metal boundaries. ${ }^{27}$ For a detailed account of the NLHD theory, we refer to Chapter 3 by N. A. Mortensen et al. The description of electron tunneling requires a quantum treatment beyond classical electrodynamics. Such approaches become available nowadays, and the optical response of plasmonic nanostructures can be calculated either extending the classical theory to incorporate electron tunneling, or by adopting a full quantum treatment using e.g. the time dependent density functional theory (TDDFT) combined with ab initio atomistic or model free-electron descriptions of metal nanoparticles. ${ }^{28-39}$ Besides properly accounting for electron tunneling, the TDDFT calculations reveal a sensitivity of the near and far fields to the fine details of the atomistic structure inside the gap between plasmonic 
nanoparticles, such as vertices and edges at contact of different crystallographic planes.

Later in this chapter, we present a detailed account of the theoretical developments based on the full quantum TDDFT calculations and discuss related recent experiments. It is noteworthy that owing to the extremely strong field enhancement in the narrow gap between plasmonic nanoparticles, the plasmonic dimer appears as the system of choice to address quantum effects and will be often quoted in this chapter.

\subsection{Non-local Screening}

Non-locality of the screening results from the collective quantum behavior of the interacting many-electron system and reflects the fact that the response of the conduction electrons at a particular point in space depends not only on the field applied at that point but also on the fields applied at other different points. For a monochromatic incident electromagnetic wave with frequency $\omega$, one can write $\mathbf{P}(\mathbf{r}, \omega)$, the polarization of the medium at point $\mathbf{r}$, as:

$$
\mathbf{P}(\mathbf{r}, \omega)=\int \mathrm{d} \mathbf{r}^{\prime} \chi\left(\mathbf{r}, \mathbf{r}^{\prime}, \omega\right) \mathbf{E}\left(\mathbf{r}^{\prime}, \omega\right),
$$

where $\mathbf{E}\left(\mathbf{r}^{\prime}, \omega\right)$ is the electric field, and $\chi\left(\mathbf{r}, \mathbf{r}^{\prime}, \omega\right)$ is the susceptibility of the medium.

Contrary to local classical descriptions, the non-local effect and the spill out of the valence electron density outside the metal prevents a sharp localization of the screening charges at the boundaries of the interfaces. The screening charges thus have finite spatial extension, as we illustrate in Fig. 4.1, where the results of the TDDFT calculation of the electron density dynamics triggered by an external laser pulse incident at a sodium nanowire dimer in vacuum are shown. ${ }^{33,34}$ Sodium is a typical free-electron metal for which the jellium metal (JM) approximation applies well. Within the JM model, the ionic cores are not explicitly introduced into the calculation, but they are replaced by a uniform positive background charge with density $n_{+}=\left[4 \pi r_{\mathrm{s}}^{3} / 3\right]^{-1}$. The Wigner-Seitz radius is $r_{\mathrm{s}}=2.12 \AA$ for Na. The 




(a)

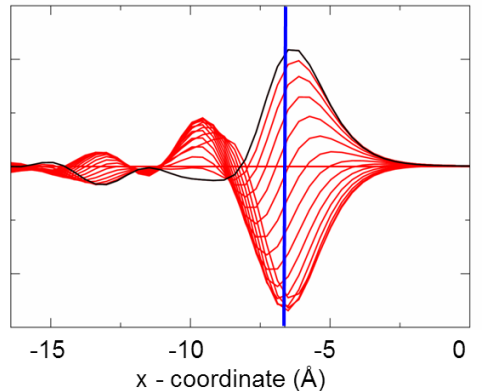

(b)

Fig. 4.1. Dynamical screening. (a) Sketch of the geometry of a nanowire dimer. Two identical cylindrical nanowires are infinite along $z$-axis and have a diameter $D=2 R_{\mathrm{cl}}$. The nanowires are separated by a gap of width $S$. The incident radiation is linearly polarized with the electric field along the dimer axis ( $x$-axis). (b) Evolution of the density induced by an incident field at the surface of the left cylinder facing the junction. The data are shown as a function of the $x$-coordinate for $D=9.8 \mathrm{~nm}$, and $S=1.3 \mathrm{~nm} . x=0$ is at the center of the junction. Different curves correspond to instants of time spanning $1 / 2$ optical period starting from $t_{0}$ (black curve). The frequency of the incoming $x$-polarized electromagnetic wave is in resonance with the bonding dipole plasmon mode at $\omega_{\mathrm{dp}}=3.157 \mathrm{eV}$. The vertical blue line denotes the position of the jellium edge.

boundary between the background charge density $n_{+}$and surrounding medium is set at half of the spacing between atomic planes in the direction of the surface. This boundary, also called "jellium edge", will be considered here as the geometrical surface of the metal.

While in the local classical approach the screening charges reside at the geometrical surfaces of the cylinders separated by the junction of width $S$, the shape of the induced charge density obtained in TDDFT calculations shows a pronounced time-dependence in the finite spatial profile. In particular, because of the spill-out effect, the induced charge density extends outside the metal boundaries by some $\AA$. One can then define the real part of the centroid of the induced charge density (Feibelman parameter) $\operatorname{Re}[d(\omega)]$ as a measure of the actual position of the plasmon-induced surface charges with respect to the jellium edge. ${ }^{20,21,40-42}$ The Feibelman parameter plays a central role in the theory of dynamic screening at surfaces and allows to describe the dispersion of propagating surface plasmons 
and finite size effects in localized nanoparticle plasmons, as we will show below. For the present case of sodium nanowires, $\operatorname{Re}[d(\omega)]$ is positive $\approx 0.9 \AA$ in the frequency range of interest. ${ }^{42}$ This means that the screening charge is shifted by $0.9 \AA$ into the vacuum and located at $\approx 3 \AA$ outside the surface atomic plane of the nanoparticle.

One of the most prominent manifestations of the non-local screening is the nanoparticle size-dependent shift of the frequency $\omega_{\mathrm{dp}}$ of the localized nanoparticle plasmon with dipolar character, as has been thoroughly studied in the case of extinction resonances in cluster physics. ${ }^{2,20,23,41,43}$ For nanoparticles characterized by a radius $R_{\mathrm{cl}}$, comparable with the wavelength of the incident radiation $\lambda$, retardation effects lead to a redshift of the localized dipolar plasmon frequency. As the size of the nanoparticles decreases, the solution of classical Maxwell's equations predicts that $\omega_{\mathrm{dp}}$ converges to a constant value $\omega_{\text {Mie }}$ (Mie frequency) as $\omega_{\mathrm{dp}}=\omega_{\text {Mie }}\left(1-A\left[R_{\mathrm{cl}} / \lambda\right]^{2}\right),{ }^{44}$ where $A$ is a constant. However, for nanoparticles of nanometer scale size, the classical description is no longer applicable, and $\omega_{\mathrm{dp}}$ is determined by the electron many-body screening at the nanoparticle surface. ${ }^{20,41}$

$$
\omega_{\mathrm{dp}} / \omega_{\mathrm{Mie}}=1-\operatorname{Re}\left[d\left(\omega_{\mathrm{dp}}\right)\right] / R_{\mathrm{cl}}+O\left(R_{\mathrm{cl}}^{-2}\right) .
$$

For alkali metals and aluminum, $\operatorname{Re}\left[d\left(\omega_{\mathrm{dp}}\right)\right]$ is positive. Thus, with decreasing cluster size, the plasmon resonance redshifts from the Mie value $\omega_{\text {Mie }}$ for small size clusters. ${ }^{45-49}$ For noble metals such as $\mathrm{Au}$ and $\mathrm{Ag}$, the final size effects lead to a blue shift of the dipolar plasmon resonance with decreasing cluster size. ${ }^{16,43,50-54}$ The sheer contrast between alkali and noble metals is due to the localized $d$-electron contribution to the total screening in the latter case. ${ }^{41}$ The $d$-electrons reside close to the atomic planes beyond the jellium edge determined by the extension of the wave functions of the delocalized $s-p$ electrons. When the $d$-electron contribution to the screening is accounted for, $\operatorname{Re}[d(\omega)]$ turns negative indicating that the screening charge is predominantly induced inside the metal. 


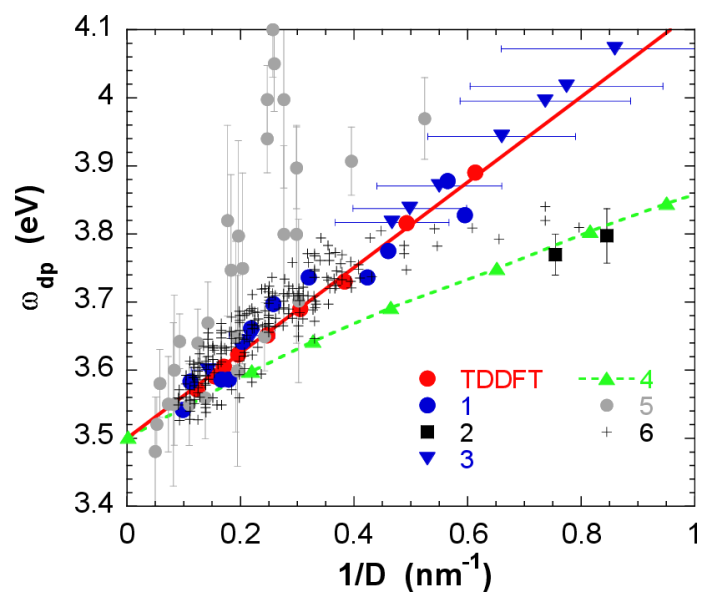

Fig. 4.2. Plasmon energy of Ag clusters as a function of the inverse of the cluster diameter $1 / D$. The dipolar bulk limit (Mie plasmon) is at $3.5 \mathrm{eV}$. The experimental data (1-5) have been scaled to obtain the resonance energies in vacuum. ${ }^{43}$ The horizontal error bars on the data (3) give the widths of the size distributions of the corresponding Ag particles. Red line with red circles shows the results of the TDDFT calculations ${ }^{55}$ using the jellium model with a polarizable background allowing to account for the $d$-electron contribution. Blue circles (1) - experimental data from Ref. [50]; Black squares (2) - experimental data from Ref. [51]; inverted blue triangles (3) - experimental data from Ref. [52]; green triangles (4) - experimental data from Ref. [53]; gray circles (5) - experimental data from Ref. [16]; (6) - data obtained in the STM induced light emission experiments. ${ }^{54}$

The difference between free electron (alkali and $\mathrm{Al}$ ) and noble metals is illustrated with Figs. 4.2 and 4.3. Measured and calculated cluster size dependence of the dipolar plasmon resonance frequency is shown for silver and sodium clusters, respectively. For the theoretical calculations, the jellium model of the metal has been used in both cases. In the TDDFT study of the optical response of Ag clusters, the polarizable background with radius $R_{\mathrm{d}}$ has been introduced inside the cluster to model the contribution of the localized $d$-electrons to the screening. ${ }^{36,56}$ The polarizable background is described by the non-dispersive dielectric function $\varepsilon_{d}=4.58$, and it has a radius defined as $R_{\mathrm{d}}=R_{\mathrm{cl}}-1.2 \AA$. As follows from the Figs. 4.2 and 4.3, and in agreement with the general discussion based on the Feibelman parameter, $\mathrm{Ag}$ and $\mathrm{Na}$ clusters display opposite finite size effect. It is 


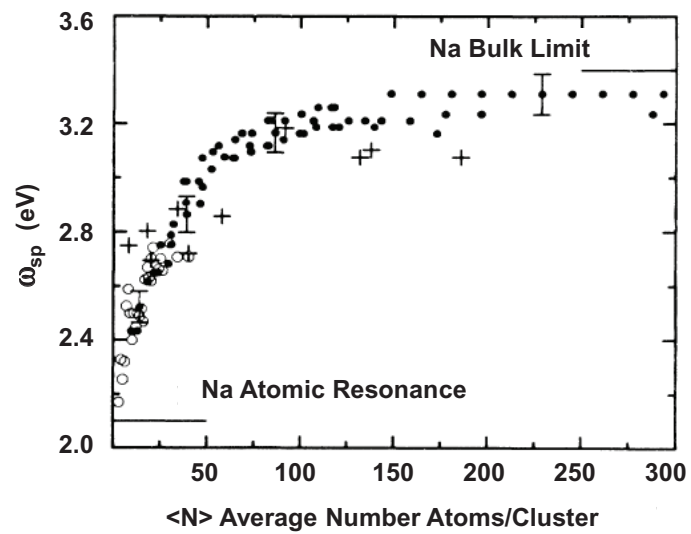

Fig. 4.3. From Ref. 47, energy of the dipolar resonance of $\mathrm{Na}$ clusters as a function of the average number of atoms $\langle N\rangle$ forming the cluster. Frequencies are derived from experimental data (solid and open circles) and compared with theoretical calculations ${ }^{45}$ (circles) based on the free-electron description of $\mathrm{Na}$.

worthwhile to stress the good agreement between the experimental data and the theoretical results based on the jellium description of the metal. This indicates that the JM captures the main physics of the screening as recently confirmed by quantum studies that adopt a full atomistic description of the metal nanoparticles. ${ }^{37,57-61}$ As appears from the discussion above, the dynamical surface screening is the main mechanism determining the size dependence of the energy of the localized plasmons in small metallic spherical nanoparticles. In a recent work, R. C. Monreal, T. J. Antosiewicz and S. P. Apell show that for quantum confinement effects linked with the quantization of the electronic states to become noticeable the nanoparticle size has to be in the $1 \mathrm{~nm}$ range and below. ${ }^{23}$

Dynamic screening not only affects the energies of the plasmon modes in individual nanoparticles, but it also does affect plasmon hybridization in adjacent metallic nanostructures. The plasmonic dimer, ${ }^{33,34}$ a canonical coupled structure, allows for a clear cut physical interpretation of quantum effects in the optical response. Fig. 4.4 presents the analysis of the role played by dynamic screening in determining the frequency of the bonding dipole plasmon, 


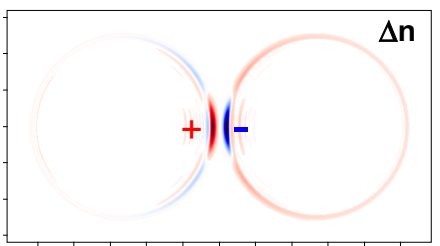

(a)

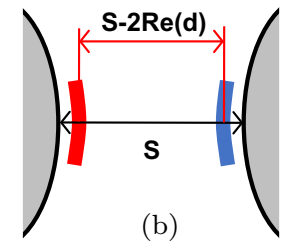

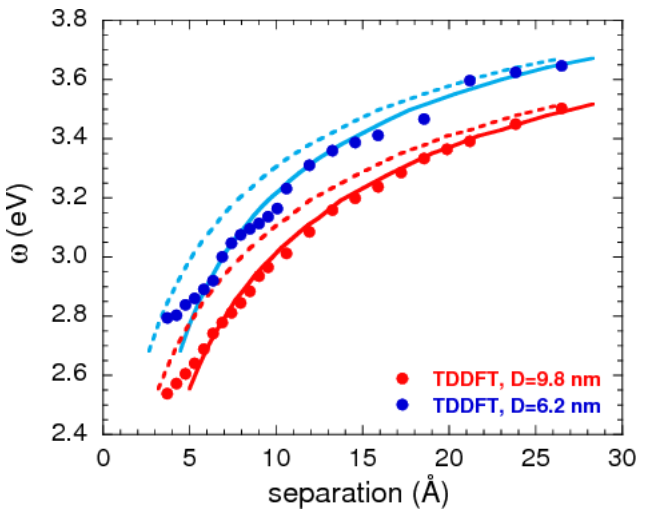

(c)

Fig. 4.4. Dynamic screening in a cylindrical nanowire dimer with $D=9.8 \mathrm{~nm}$ and $S=0.8 \mathrm{~nm}$, as sketched in Fig. 4.1. (a) The TDDFT result for the charge density $\Delta n$ induced by the incident $x$-polarized laser pulse at resonance with the lowest bonding dipole plasmon mode of the system $\left(\omega_{\mathrm{dp}}=3.1 \mathrm{eV}\right)$. Positive (negative) values correspond to red (blue) color code. $\Delta n$ is shown at the instant of time corresponding to the maximum dipole moment of the dimer. (b) Zoom into the junction with schematic representation of the location of the plasmon-induced screening charges. Within the local classical approach, the screening charges are exactly located at the geometrical surfaces of the cylinders (here equivalent to the jellium edges) separated by the junction of width $S$. As calculated with TDDFT, the centroids of the screening charges (red area for positive charge and blue area for negative charge) are located at $\operatorname{Re}[d(\omega)]$ in front of the jellium edges, and therefore separated by $S-2 \operatorname{Re}[d(\omega)]$. (b) Energy of the dipole plasmon resonance as a function of the junction width $S$. Dots: TDDFT results obtained for nanowire dimers formed by $D=6.2 \mathrm{~nm}$ (blue) and $D=9.8 \mathrm{~nm}$ (red) individual nanowires. The results of classical Drude calculations for $D=6.2 \mathrm{~nm}$ and $D=9.8 \mathrm{~nm}$ dimers are shown, respectively, with blue and red lines. Dashed/solid line type: separation $S$ is measured between the jellium edges/centroids of the induced charges. For more details, see the main text.

also known as gap plasmon. Panel (a) shows the TDDFT result for the charge density induced by a $x$-polarized laser pulse incident on the $D=9.8 \mathrm{~nm}, S=0.8 \mathrm{~nm}$ nanowire dimer sketched in Fig. 4.1. The incident radiation is at resonance with the bonding dipolar plasmon of the dimer formed by the hybridization of the plasmon dipolar modes of the individual nanowires. The systems with narrow gaps are characterized by a strong field enhancement in the junction because 
of the large charge densities of opposite sign induced by the plasmon at the facing metal surfaces across the gap [see panel (a)]. In turn, this results in an attractive interaction leading to the redshift of the energies of the bonding plasmon modes of the dimer as compared to those of the individual particles. In the classical theory, the field enhancement and the redshift diverge for a vanishing width of the junction $S \rightarrow 0 .{ }^{62}$ The "zero width catastrophe" is removed when the electron tunneling is accounted for as we discuss in the next section. At present, we limit the discussion to $S>0.5 \mathrm{~nm}$, where the tunneling effect is negligible, and the induced charge densities can be well assigned to the individual cylinders forming the junction.

We recall that within TDDFT, the real part of the centroid of the induced charge density $\operatorname{Re}[d(\omega)]$ corresponds to the position of the plasmon-induced surface charges with respect to the jellium edge of each cylinder. For the present case of sodium nanowires, $\operatorname{Re}[d(\omega)] \approx 0.9 \AA$ with screening charge shifted from the jellium edge into the vacuum. As we schematically show in panel (b) of Fig. 4.4, for a junction with width $S$ measured between the jellium edges, the actual distance between the plasmon-induced charges across the junction is $\Sigma=S-2 \operatorname{Re}[d(\omega)]$. This insight provides an explanation of the dependence of the energies of the bonding dipolar plasmon as a function of gap width, as shown in Fig. 4.4(c) for two different nanowire dimers. ${ }^{34}$ While classical electromagnetic calculations reproduce the TDDFT data overall, the latter is systematically redshifted with respect to the former. Indeed, in the classical theory, the screening charges are confined at the metal surfaces separated by the junction of width $S$, while in the quantum theory, the actual distance between the screening charges $\Sigma$ is smaller leading to a stronger interaction (dots versus dashed lines in Fig. 4.4(c)). Thus, the TDDFT data obtained for a junction with a specific width $S$ (dots) should be compared with the classical results calculated for a gap of effective width $\Sigma=S-2 \operatorname{Re}[d(\omega)]$ (solid lines). In doing so, the agreement between TDDFT and the classical simulations becomes excellent indicating that in this case the effect of the non-local screening can be accounted for within the classical theory by simple distance 
rescaling. ${ }^{33,34}$ The distance rescaling provides an estimate of the characteristic gap sizes $S$, for which the non-local screening is important. Indeed, the shift of the plasmon energy from the classical value starts to be noticeable for $2 \operatorname{Re}[d(\omega)] / S \sim 0.1$. Provided that the Feibelman parameter $\operatorname{Re}[d(\omega)]$ is in the $\AA$ range for typical metals, we obtain $S \leq 2-3 \mathrm{~nm}$. Note that for small widths of the gap $S<0.5 \mathrm{~nm}$, the electron densities of the individual nanowires overlap indicating the onset of efficient tunneling which then dominates the optical response (see next section).

In addition to an explanation of the change of energies of the localized nanoparticle plasmons, the dynamic surface screening theory can be also applied to describe the cluster size dependence of the excited electron dynamics in the system. Indeed, via many-body interactions, the collective electron excitation (plasmon) decays into an incoherent excitation of electron-hole pairs. ${ }^{2,21,22,56,63-66}$ The surface contribution to this Landau damping mechanism can be described via the imaginary part, $\operatorname{Im}[d(\omega)]$, of the Feibelman parameter. ${ }^{21}$ For the sake of clarity, we will use much simpler phenomenological description here. ${ }^{65}$ The width of the localized plasmon resonance $\Gamma$ (inverse of the lifetime of the collective mode $\tau=1 / \Gamma$ ) is given by several contributions:

$$
\Gamma=\Gamma_{\mathrm{rd}}+\Gamma_{0}+g_{\mathrm{s}} v_{\mathrm{f}} / R_{\mathrm{cl}} .
$$

The first term, $\Gamma_{\mathrm{rd}} \propto R_{\mathrm{cl}}^{3}$, stands for the radiative decay rate. ${ }^{66,67}$ The second term gives the bulk contribution to the Landau damping and the last term describes the surface contribution. The latter mainly introduces a decay rate which is proportional to the frequency of the collisions with the surface $v_{\mathrm{f}} / R_{\mathrm{cl}}$ of a classical electron moving with Fermi velocity $v_{\mathrm{f}}$. As follows from Eq. (4.3), for large nanoparticle sizes, radiative decay dominates; however, for small nanoparticles the incoherent electron-hole excitation becomes a leading mechanism with the surface contribution increasing as $1 / R_{\mathrm{cl}}$. This is further illustrated in Fig. 4.5, where it can be observed that as a silver nanoparticle size decreases, the spectral width, $\Gamma$, of the plasmon resonance shows a transition from a radiative decay regime to a regime of decay via electron hole-pair excitation. 


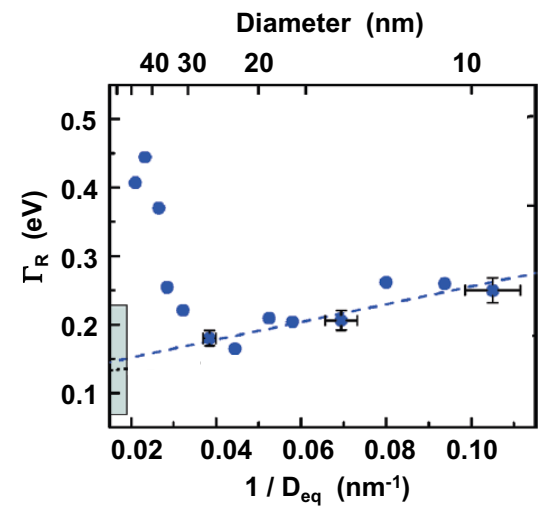

Fig. 4.5. From Ref. 65, spectral width $\Gamma$ of the localized surface plasmon resonance measured in different single $\mathrm{Ag} / \mathrm{SiO}_{2}$ nanoparticles as a function of the inverse of their equivalent diameter $D_{\text {eq }}$, optically determined by fitting the extinction spectra. Error bars on size determination and width are indicated for three typical sizes. The blue dashed line is a linear fit using Eq. (4.3) and assuming negligible radiative decay $\Gamma_{\mathrm{rd}}=0$, with $g_{s}=0.7$ and $\Gamma_{0}=0.125 \mathrm{eV}$. The shaded area indicates the estimated $\Gamma_{0}$ value in bulk silver.

While transfer of the electromagnetic energy trapped by the localized plasmon to the electron-hole excitations in the metal is one of the main bottlenecks in using plasmonic materials for information technology, this effect is extremely beneficial for many applications induced by hot electrons such as local heating, photovoltaics, nanoscale detectors and photochemistry at surfaces. ${ }^{10,68-71}$

\subsection{Electron Tunneling}

Electron tunneling through the potential barrier separating classically allowed regions of motion is one of the prominent quantum features that cannot be captured by the classical theory. This effect forms the basis of a powerful method of surface analysis, namely scanning tunneling microscopy (STM) ${ }^{72}$ where a tunneling current across the junction formed by a STM tip and a surface is triggered by an applied external DC bias. In the $\mathrm{AC}$ regime, photon-assisted tunneling in metal-dielectric-metal or semiconductor junctions has been studied in the $\mathrm{THz}$ range. ${ }^{24,25}$ However, it is only recently that 
full quantum theories and experimental studies performed with wellcontrolled junctions addressed tunneling phenomena in plasmonics at optical frequencies. ${ }^{15-19,28,30-38,73-79,82-84}$ Electron tunneling, or, in broader terms, electron transfer between nanoparticles and its interaction with photons coupled to plasmonic excitations is at the origin of many physical phenomena in narrow gaps. To access the tunneling regime in plasmonics allows (i) to understand the transition from separated nanoparticles to touching particles, when the gap sizes are of the order of the distances between atomic planes in the metal, (ii) to design nanoscale electro-optical devices capable of either generating electronic currents with incident photons or producing electroluminescence due to inelastic tunneling of electrons, and (iii) to develop strategies for controlling the plasmon response such as those offered by functionalization of the junction with self-assembled molecular layers (SAMs).

Because of the difficulty to reach reproducible sub-nanometer gaps in experimental conditions, the tunneling effect in plasmonics was first studied in nanoparticle dimers theoretically. ${ }^{28,30,31}$ These theoretical predictions have been followed by experimental verification of the tunneling regime. ${ }^{15-19,73-76}$ In Fig. 4.6 we show the optical response of a $D=9.8 \mathrm{~nm}$ jellium Na nanowire dimer in vacuum (Fig. 4.1). The results are obtained using different, classical, quantum and mixed, approaches not allowing or allowing to account for the electron transport through plasmonic gap. ${ }^{34}$ Similar results have been reported for a dimer formed by two spherical nanoparticles represented using the free-electron $\mathrm{JM},{ }^{28,30,31}$ or described at full atomistic level. ${ }^{37}$ Thus, one can identify general and robust features of the modification of the optical response due to the electron transfer between nanoparticles.

A comparison between the quantum TDDFT results and the results from the classical electromagnetic calculations reveals several important differences as the junction width $S$ is decreased:

- The redshift of the energies of the plasmon modes as calculated with TDDFT is finite, not diverging as in the classical theory. 



Fig. 4.6. Optical response of a $\mathrm{Na}$ nanowire dimer in vacuum. The diameter of each nanowire is $D=9.8 \mathrm{~nm}$, as depicted in Fig. 4.1. Results are obtained with the TDDFT calculations (TDDFT), with classical Drude electromagnetic calculations (Drude), and with the quantum corrected model (QCM). The latter allows to account for electron transport across the junction within the framework of classical electrodynamics. The incoming plane wave is polarized along the dimer axis $x$. Upper panels: Waterfall plots of the dipole absorption cross-section per unit length, $\sigma$, as a function of the width of the junction $S$ and frequency $\omega$ of the incident radiation. The centers of the wires are at $x= \pm(D+S) /(2)$, and $S$ is negative for overlapping cylinders. For clarity, a vertical shift proportional to $S$ is introduced for each absorption spectrum. The red curves are used for a change of $S$ every $2.65 \AA$. These curves are labeled with the corresponding $S$-values every $5.3 \AA$. The plasmonic modes responsible for the peaks in the absorption crosssection are labeled: Bonding Dipole Plasmon (DP), Bonding Quadrupole Plasmon 
Fig. 4.6. (caption continued) (QP), high-order hybridized mode (HM), lowest (dipole) Charge Transfer Plasmon (C1), and higher energy Charge Transfer Plasmon (C2). Lower panels: Color plots of the local field enhancement at the center of the junction for positive separations. Results are shown as a function of the frequency $\omega$ of the incident radiation and junction width $S$. The color code is displayed at the bottom of the corresponding panels.

- The number of plasmon resonances is much smaller in the TDDFT results.

- The bonding plasmon modes of the separated dimer disappear, and the charge transfer plasmon modes ${ }^{89}$ emerge in the TDDFT results prior to direct geometrical contact between the nanowire surfaces at $S=0$.

- The field enhancement in the middle of the junction, as obtained with TDDFT, stays finite and it is quenched for small $S$ in sheer contrast with the divergence of the fields observed from the classical description.

All over, the transition from a capacitively $(S>0)$ to a conductively $(S \leq 0)$ coupled system is not discontinuous as in classical calculations, but continuous. This is because the electron tunneling between metal surfaces separated by the narrow gaps leads to the establishment of a conductive contact across the junction prior to the direct geometrical touch between the surfaces. Indeed, in the local classical calculations the divergence of the shifts of the plasmon modes energies and the field enhancement at vanishing gap width results from the divergent coulomb interaction between the screening charges of opposite sign localized at the geometrical surfaces across the junction. In the quantum calculations, however, the tunneling current neutralizes the plasmon-induced charges and thus reduces the field enhancement and the shift of the plasmon modes energies. This tunneling current also leads to the progressive attenuation of the bonding plasmon modes of the dimer formed by the hybridization of the plasmon modes of the individual nanoparticles. From the point 
of view of the underlying physics, accounting for the ability of conduction electrons to tunnel through the potential barrier separating two nanoobjects allows to correctly capture the transition from the the situation of disconnected nanoparticles to the situation with the continuous metal bridge linking the junction.

Recently, quantum effects in the optical response of metallic nanoparticles have been addressed with computationally involved calculations based on a full atomistic description of the nanoparticles. ${ }^{37-39}$ Undoubtedly, the atomistic structure of the nanoparticles leads to a quantitative dependence of the near fields and optical cross-sections on particular morphological aspects of the surfaces, e.g. specific surface facets forming the junction. The formation of atomicsize hot spots at the vertices and edges between crystallographic planes has been also reported. ${ }^{39,85}$ Nevertheless, the general trends of the plasmon modes evolution in narrow gaps derived from TDDFT calculations within the JM description of the nanoparticles hold.

More insights into the effect of electron tunneling across the junction can be gained by analyzing the dynamics of currents and charges in the nanowire dimer induced by an incident $x$-polarized laser pulse resonant with the lowest (DP or $\mathrm{C} 1$ ) plasmonic modes, as displayed in Fig. 4.7. For a wide junction with $S=7.95 \AA$, the total induced dipole is formed by the in-phase dipolar polarization of each nanocylinder, as expected for the bonding DP mode. The maximum of the dipole is reached at the instant of time when the maximum charge separation has occurred and the currents inside the nanoparticles are minimal. In that situation, high charge densities are induced at the surfaces facing the junction, resulting in large electric field enhancement. The structure of the induced charges and fields is similar to previous classical results for coupled cylinders ${ }^{86,87}$ or metal spherical dimers. ${ }^{62}$ For such large separations, the probability of tunneling between nanowires is negligible. When the junction width is reduced to $S=2.65 \AA$, basically the same profiles for induced densities and fields are obtained. However, in this situation, opticallyassisted AC tunneling current between the nanoparticles can now be 


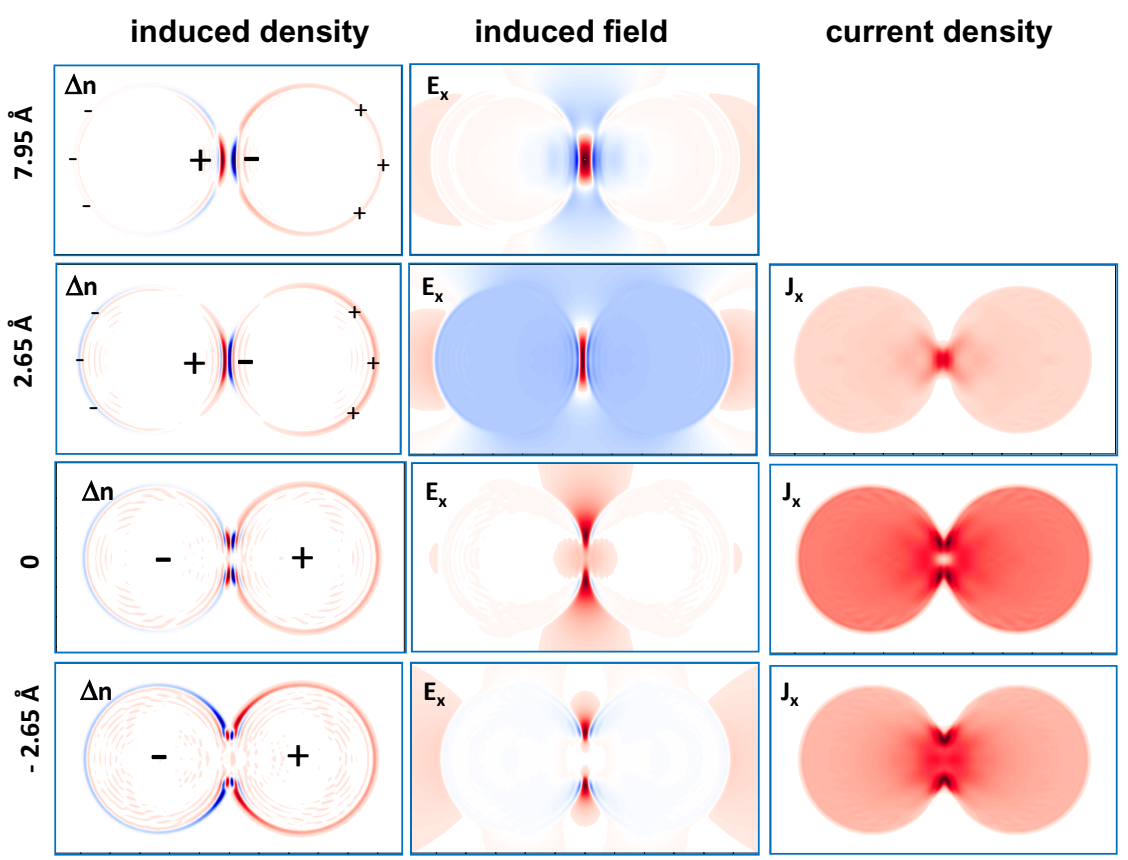

Fig. 4.7. Plasmon dynamics in a $D=9.8 \mathrm{~nm}$ nanowire dimer system. The incident $x$-polarized laser pulse is at resonance with the lowest bonding dipolar plasmon DP for $S>0$, or with the lowest charge transfer plasmon $\mathrm{C} 1$ for $S \leq 0$. Panels in the left column: snapshots of the induced charge density $\Delta n$; middle column: snapshots of the electric field along the $x$-axis $E_{x}$; right column: current density $J_{x}$ along the $x$-axis. Positive (negative) values correspond to the red (blue) color code. Results within each row correspond to a fixed junction width $S$, as indicated to the left of each row. The induced densities are shown at the instant of time corresponding to the maximum dipole moment of the dimer. The induced currents and fields are shown at the instants of time when the induced fields in the junction reach the maximum. $J_{x}$ is not shown for the $S=7.95 \AA$ because the tunneling current is negligible in that case.

observed..$^{24,25,80,81}$ The junction shows a resistive character ${ }^{24}$ with the maximum tunneling current between nanowires reached at the maximum field and consequently at the maximum induced dipole. Because of the tunneling, the field enhancement in the junction is reduced (see Fig. 4.6). 
Further reduction of the junction width $S$ increases the tunneling probability and short-circuits the junction. When the conductive contact is formed, the fields are expelled from the junction, ${ }^{32,88}$ the DP mode disappears and the charge transfer modes emerge in the absorption spectrum. In this respect, the results obtained for $S=0$ and $S=-2.65 \AA$ are very similar. The maximum dipole moment of the dimer now corresponds to oppositely charged nanowires. The maximum currents and fields are reached when the total dipole moment of the system is minimum. Indeed, the current flowing trough the junction builds the dipole polarization with opposite charges at the left and right nanowires.

The theory of electron tunneling in the presence of a DC electromagnetic field ${ }^{24,25,80,81}$ provides a formal framework that allows to describe the evolution of the plasmon response in nanoparticle assemblies with narrow gaps. Assuming linear response regime, the optical field $E_{\omega}$ at frequency $\omega$ induces a tunneling current at the same frequency in the junction between two flat metal surfaces. The dissipative component (in phase with the driving field) of this AC tunneling current density is given (in atomic units) by: ${ }^{24,83}$

$$
J_{\omega}=\frac{V_{\omega}}{2 \omega}\left[J_{\mathrm{DC}}(U+\omega)-J_{\mathrm{DC}}(U-\omega)\right],
$$

where $U$ is the applied DC bias, $V_{\omega} \simeq \mathrm{SE}_{\omega}$ is the (small) optical bias across the junction, and $J_{\mathrm{DC}}(U \pm \omega)$ is the DC tunneling current at the constant bias $U \pm \omega$. Here, we assume that $E_{\omega}$ does not vary across the junction. Equation (4.4) establishes a connection between the AC tunneling characteristics of the junction and the static DC tunneling characteristics, commonly used in the description of STM. ${ }^{81}$ For slow variation of $J_{\mathrm{DC}}$ with $U$, we obtain the classical limit $J_{\omega}=\mathrm{SE}_{\omega} \mathrm{d} J_{\mathrm{DC}}(U) / \mathrm{d} U$, which can be written as

$$
\begin{aligned}
J_{\omega} & =G(U, S) V_{\omega}=\sigma(U, S) E_{\omega}, \\
G(U, S) & =\mathrm{d} J_{\mathrm{DC}}(U) / \mathrm{d} U, \\
\sigma(U, S) & =S G(U, S),
\end{aligned}
$$


where $G(U, S)$ is the DC conductance of the junction at bias $U$, and $\sigma(U, S)$ is the DC condutivity of the junction. One expects that the tunneling current starts affecting the plasmon modes when it allows partial neutralization of the plasmon-induced screening charge densities $\varrho$ at the metal surfaces across the junction. ${ }^{89}$ This implies that $J_{\omega} \simeq \mathrm{d} \varrho / \mathrm{d} t$. Assuming $J_{\omega}=G(U, S) \mathrm{SE}_{\omega}$, and $E_{\omega}=4 \pi \varrho$ (flat capacitor), the harmonic time dependence of the fields results in $G(U, S) \mathrm{SE}_{\omega} \simeq \omega E_{\omega} / 4 \pi$. Thus, the value of the conductance of the junction at which tunneling effects are noticeable on the plasmon modes can be estimated from:

$$
G(U, S)=\omega / 4 \pi S
$$

For a junction of width of $0.5 \mathrm{~nm}$, and $\omega \approx 3 \mathrm{eV}$, Eq. (4.6) results in $G(U, S) \approx 2 \times 10^{-3} G_{0}$, where $G_{0}=7.75 \times 10^{-5} \mathrm{~S}$ is the quantum of conductance. This estimate is consistent with the range of conductance values reported for the onset of quantum effects on the bonding plasmon modes of dimer structures within the TDDFT calculations in the absence of applied bias $(U=0) .{ }^{30-34,38,39,90}$ Since $G(U, S)$ increases exponentially with decreasing junction width, a variation of $S$ is the most efficient way to enter into the quantum regime. However, a modification of the externally applied bias might provide an additional degree of freedom to tune the optical response of the nanostructure, together with the functionalization of the plasmonic gaps with molecular layers, as discussed below.

The approach expressed by Eqs. (4.4) and (4.5) is based on the theory of photon-assisted tunneling, it describes the resistive AC tunneling currents, and thus it can be applied in the case of the junctions with low tunneling probabilities and resistive character. This approach can thus explain the attenuation of the bonding plasmon modes of the dimer (DP, QP) (see Fig. 4.6). However, it does not allow to describe the evolution of the junction from resistive to conductive upon further decreasing $S$, as shown in Fig. 4.8. Panel (a) of the figure illustrates the evolution of the electron tunneling barrier across the junction between two metal surfaces when the junction width is reduced from $S_{1}$ to $S_{2}$, where $S_{2}<S_{1}$. Panel (b) displays 
the time-evolution of the current density $J_{x}(t)$ across the middle of the junction between two $D=9.8 \mathrm{~nm}$ metal cylinders. $J_{x}(t)$ is shown as a function of the electric field $E_{x}(t)$ at the same position. The subscript refers to the projection on the x-axis of the dimer (see Fig. 4.1(a)). The time dependence of the currents and fields is calculated within TDDFT for an incident plane wave resonant with the corresponding plasmon mode of the system (see caption). If the frequency-dependent conductivity of the junction is defined as

$$
\sigma(S, \omega)=J_{x}(\omega) / E_{x}(\omega)
$$

then the linear proportionality between $J_{x}(t)$ and $E_{x}(t)$ with no relative phase, as shown in panel (b) of Fig. 4.8 for large $S$ (black lines), implies that $\sigma(S, \omega)$ is real valued (resistive junction). This conductivity can be obtained from the TDDFT results, as shown above, or it can be approximated by its DC value defined by Eq. (4.5), since the approach developed to describe tunneling conductances $G$ in STM junctions ${ }^{31,91}$ can be applied. At small $S$, the relative phase between $J_{x}(\omega)$ and $E_{x}(\omega)$ implies that $\sigma(S, \omega)$ is complex. Thus, the junction character evolved from resistive [large $S$ ] to conductive [small $S$ ]. The tunneling barrier is low in that situation, and electrons are efficiently transferred between the nanoparticles. The conductance of the junction is in the $0.1-1 G_{0}$ range, which is the threshold for observing the emergence of charge transfer plasmon modes. ${ }^{38,39,89,92}$ Obviously, at $S=0$ the potential barrier vanishes and the metal-to-metal contact is formed.

The analysis of the quantum regime in tunneling junctions and its consequences can be summarized as follows:

- In the absence of tunneling, the non-local screening affects the energies of plasmon modes in junctions with $S$ in the range of some nanometers. This effect can be accounted for by rescaling classical results.

- For junction widths below $0.5 \mathrm{~nm}$, electron transfer between nanoparticles is a dominating process that determines the optical response. The range of the electron tunneling effect as given here, 


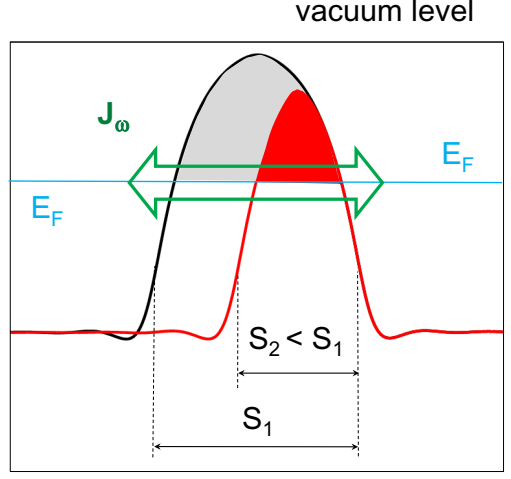

(a)

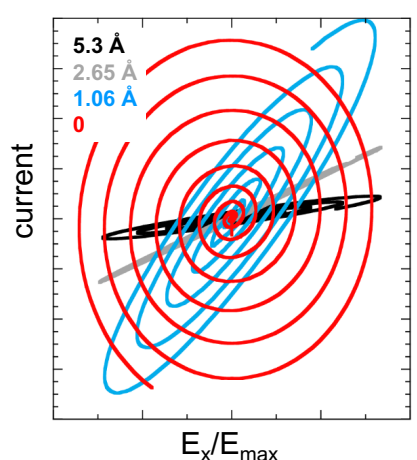

(b)

Fig. 4.8. (a). Schematic representation of the evolution of the electron tunneling barrier across a nanoparticle junction when the junction width is reduced from $S_{1}$ to $S_{2}$, where $S_{2}<S_{1}$. No bias is applied. The shaded areas represent the tunneling barrier for the electrons at the Fermi energy before (gray) and after (red) changing $S$. Green arrow shows the AC current $J_{\omega}$ due to the incident electromagnetic field at frequency $\omega$. (b) Conductivity analysis for the $D=9.8 \mathrm{~nm}$ nanowire dimer system. The current density $J_{x}$ calculated with TDDFT on the $x$-axis in the middle of the junction is plotted as a function of the normalized electric field component $E_{x}$ at the same position. Different colors correspond to different junction widths $S$ as labeled in the insert. The incident $x$-polarized laser pulse is at resonance with the lowest (DP) plasmon mode for junctions of width $S=2.65 \AA$ and $S=5.3 \AA$. For the vanishing junction, $S=0$ and $S=1.06 \AA$, the incident pulse is at resonance with the $\mathrm{C} 1$ charge transfer plasmon mode. For further details see Ref. 34 .

is only an estimate. It depends on the specific conditions and can be modified through the change of the potential barrier.

- Standard classical electromagnetic approaches do not allow to describe the electron tunneling effect.

- Quantum calculations naturally include tunneling, but being computationally demanding, are limited to systems too small as compared to those of actual interest in plasmonics.

- For not-too-narrow junctions (low $G$ ) much of the understanding of the modal description can be gained from the theory of photonassisted tunneling. However, this theory does not allow for a simple description of the transition from the tunneling to full contact regime. 
A recent proposal to cope with the issues pointed above consists in describing the electron transport properties of the junction via an effective dielectric constant. In this way, the so-called $\mathrm{QCM}^{31}$ allows to account for the tunneling between plasmonic nanoparticles within the framework of classical Maxwell's equations so that the standard efficient electromagnetic solvers can be applied in practical plasmonic morphologies of considerable size. ${ }^{15,31,34,90,93}$ The QCM approach is grounded on two observations. First, the widths of nanogaps where tunneling effects are important are much smaller than the typical curvature radii of the plasmonic nanoparticles forming the junction. The quantum effects in the nanogap can be then modelled using the properties of the electron transfer as calculated for planar metal surfaces separated by $S$, and then continuously varying $S$ according to the actual geometry of the system. Second, since $S<\lambda$ ( $\lambda$ is the wavelength at optical frequencies), Eq. (4.7) can be considered as a local one. We can thus assume that the local permittivity of the gap can be defined as

$$
\varepsilon(S, \omega)=1+i 4 \pi \sigma(S, \omega) / \omega .
$$

Applying Eq. (4.7), the conductivity $\sigma(S, \omega)$ can be obtained from quantum calculations as shown in Fig. 4.8(b) or reported in Ref. 94. Then, if this $\varepsilon(S, \omega)$ is used to describe the response of the junction in the classical Maxwell's equations, the relationship between the current density across the gap and the optical field inside the gap as obtained from these classical calculations will be the same as the one obtained from quantum calculations. Alternatively, for resistive junctions (large enough gaps), $\sigma(S, \omega)$ can be approximated by its DC value calculated from the STM theory. ${ }^{91}$

For narrow gaps at the transition from the resistive to conductive junction, the STM theories do not apply. However, the TDDFT calculations of $\sigma(S, \omega)$ can be also avoided in this case, by enforcing the transition to a continuous metal for $S=0$ with the use of a frequency-dependent permittivity of the junction described with the 
Drude model:

$$
\varepsilon_{D}(S, \omega)=1-\frac{\omega_{p}^{2}}{\omega(\omega+i \eta(S))},
$$

where $\omega_{p}$ is the plasma frequency characteristic of the metal nanoparticles. $\eta(S)$ is set by an exponential function such that for $S \rightarrow 0$, one retrieves the attenuation of the bulk metal, and for large widths of the gap, $\varepsilon_{D}(S, \omega) \rightarrow \varepsilon(S, \omega)$, given by Equation (4.8). ${ }^{90}$ Observe that for large $S$, the dielectric function becomes independent of the particular model parametrization. The QCM is shown to correctly reproduce the full quantum results of the response for various systems such as the spherical nanoparticle dimer, ${ }^{31}$ the cylindrical nanowire dimer ${ }^{34}$ (see Fig. 4.6) and core-shell plasmonic nanoparticles. ${ }^{93}$ The model has been also applied to explain a number of recent experimental observations, as we describe in Fig. 4.9. Screening of the gap plasmon signal in Electron Energy Loss Spectra, in dark-field scattering, in nonlinear signals from gaps, or in gaps functionalized with molecular layers are some of the relevant examples which make quantum effects in plasmonics evident.

\subsection{Controlling Tunneling with Molecular Layers}

The recognition that the conductance across a gap between metal nanoparticles is a key parameter determining the tunneling effect in the plasmonic response of the system allows one to elaborate a variety of strategies for controling the latter. While varying the width of the gap $S$ seems a natural choice to control the conductance across the junction, using self-assembled molecular layers (SAMs) also provides the possibility to change this conductance, and thus to decrease/increase quantum effects. Junctions functionalized with SAMs offer very promising perspectives as they allow to impact tunneling in two ways: ${ }^{95}$ via variation of the overall tunneling barrier, ${ }^{17}$ and/or via resonant transfer involving molecular orbitals. ${ }^{92,96-99}$

Examples of molecular control over the plasmon response in metal nanostructures characterized by narrow plasmonic gaps are shown in Fig. 4.10. The nanometer-thick junction between silver 


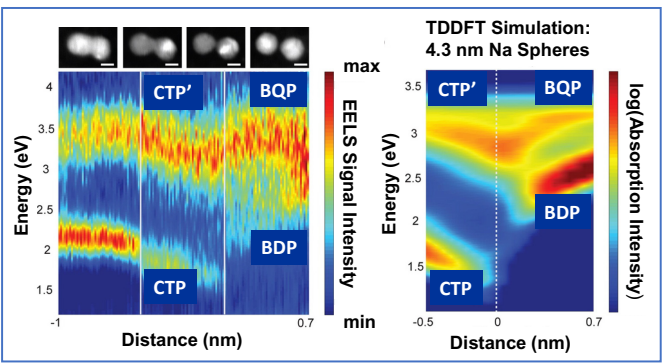

(a)

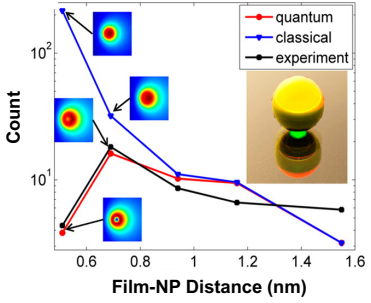

(c)

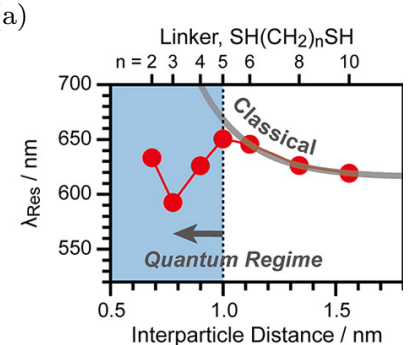

(d)

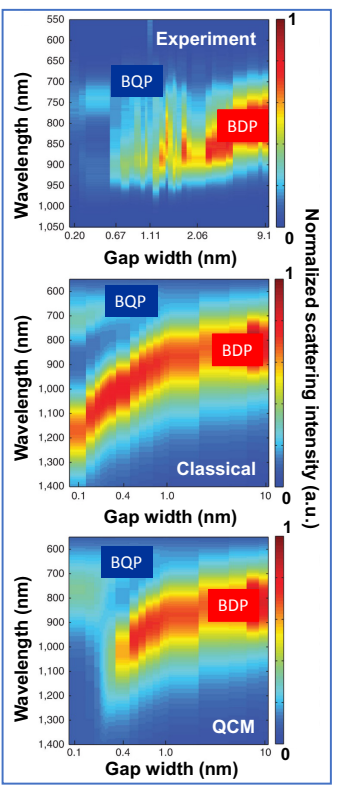

(b)

Fig. 4.9. (a) Continuous collection of EELS measured in 9-nm-diameter silver homodimer as a function of the separation distance to observe quantum tunneling effects at small gap sizes (left). ${ }^{16}$ Absorption cross-section at different separation distances calculated with TDDFT for the dimer formed by 4.3-nmdiameter sodium spheres in vacuum ${ }^{30}$ (right). BDP, BQP: bonding dipole and quadrupole plasmons, CTP, CTP': charge transfer plasmons. (b) Experiment: measured dark-field scattering spectra from gold disk dimers with various gapwidths. ${ }^{16}$ Horizontal axis represents measured gap-widths from $9.1 \mathrm{~nm}$ to $2 \AA$ in log scale. Classical: Results of the classical electromagnetic simulations of the same situation as above for gap-widths from $1 \AA$ to $10 \mathrm{~nm}$. QCM: Scattering intensities simulated with the QCM. For small gap-widths $<4 \AA$, screening of spectral features related to electron tunneling is observed and reproduced with QCM. The measured and calculated scattering intensities are normalized by their corresponding largest values. (c) Third harmonic signal as a function of the thickness of a self-assembled molecular layer separating $60 \mathrm{~nm}$ spherical gold nanoparticles from a gold surface. ${ }^{18}$ The experimental data an compared with calculations using the QCM, and the classical electromagnetic approach. Insets show the calculated electric field intensity distribution in the gold film just below the nanoparticle for a wavelength of $1570 \mathrm{~nm}$. The decrease of the nonlinear signal for small filmnanoparticle distance is attributed to the reduction of the field enhancement because of electron tunneling. (d) Evolution of the resonance wavelength of the plasmon band (red dots) 
Fig. 4.9. (caption continued) in gold nanoparticle dimers as the interparticle distance is reduced by varying the size of the molecular linker. ${ }^{75}$ The gray curve is the best fit to an exponential function, representing the classical electromagnetic model for plasmon coupling. The vertical dotted line marks an interparticle distance of $1 \mathrm{~nm}$, where quantum plasmon coupling apparently begins.

(a)

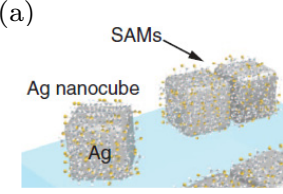

(b)



(c)

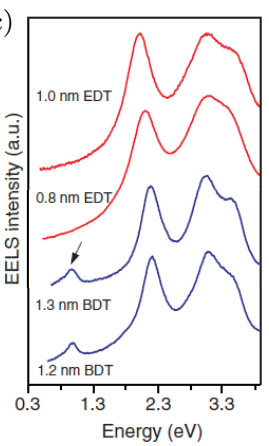

(d)



Fig. 4.10. (a)-(c) from Ref. 17. (a): Schematic illustration of the molecular tunnel junctions between two silver nanoparticles bridged by a SAM on a silicon nitride membrane. (b): The distance between two adjacent nanoparticles is determined by the thickness of the SAMs of either saturated, aliphatic 1,2ethanedithiolates (EDT) or aromatic 1,4-benzenedithiolates (BDT). (c): Measured EELS spectra for double SAMs of EDT (red) and BDT (blue). Tunneling was observed for the double-layer BDT but not in the double-layer EDT junctions. (d)-(f) from Ref. 99. (d): Conductive and non-conductive SAMs in plasmonic junctions. Schematic of a gold nanoparticle separated from a gold film by a thin molecular spacer layer. (e): Dark-field images of $60 \mathrm{~nm}$ gold nanoparticles on biphenyl-4-thiol (BPT) and biphenyl-4,4'-dithiol (BPDT), respectively. (f): Normalized scattered intensity from individual $60 \mathrm{~nm}$ gold nanoparticles on BPT and BPDT. For nearly the same dielectric constant of BPT and BPDT polymers linking the junction, tunneling leads to a blue shift of the bonding plasmon mode in the case of the BPDT film (slightly conductive) as compared to the BPD (non-conductive).

nanoparticles in one case (panels a, b and c), and between a gold nanoparticle and a gold surface in the other case (panels $\mathrm{d}$, e and f) is functionalized with SAMs. Two types of molecular layers are used in the experiments allowing to compare the results obtained with SAMs of relatively low and high conductance in order to 
reveal tunneling effects. For silver nanoparticles separated by a double molecular layer, the low-energy tunneling plasmon associated with interparticle charge transfer across the gap has been observed only for SAMs formed by higher conductance molecules [aromatic 1,4-benzenedithiolates (BDT) in this case]. For SAMs in the junction between the gold nanoparticle and the gold surface, the higher conductance biphenyl-4,4'-dithiol (BPDT) molecules induce a blue shift of the gap plasmon mode. In this case, the tunneling conditions correspond to the situation described with Equation (4.6). Tunneling current partially neutralizes the plasmon-induced screening charges at the junction interfaces, and thus reduces the attractive interaction between the nanoparticle and the substrate. ${ }^{89}$ At this point, it is important to stress that using metal-oxide-metal type of junctions also allows to change the tunneling barrier depending on the band structure of the dielectric inside the junction. Indeed, the tunneling barrier is set by the energy of the oxide conduction band minimum in this case. ${ }^{71,83,91}$ Last but not least, the examples above show that monitoring the emergence of the charge transfer plasmon and/or the blueshift of the gap plasmon provides an access to the tunneling characteristics of molecular layers. A sophistication of the connection between the conductance and the plasmonic response in SAMs could serve as a valuable tool to quantitatively measure molecular conductance at $\mathrm{AC}$ conditions.

\subsection{Conclusions}

In this chapter, we have described quantum effects in nanoplasmonics derived from the quantum nature of the many-body interactions between conduction electrons in metals. The presence of surfaces of metallic objects intrinsically brings up the effects of confinement, of dynamical screening of electromagnetic fields, of electron spill out, and of the atomistic arrangement at the interface between different materials. Plasmonic structures are key building blocks in nanophotonics where all these quantum effects can be tested. In particular, geometries characterized by narrow gaps between metallic surfaces (plasmonic gaps) emerge as canonical systems where optics and electron transport 
can be connected with unprecedented levels of control and monitoring capability. As experimental architectures reach the subnanometric scale, accounting for quantum effects becomes progressively relevant for a correct interpretation of data obtained from optical spectroscopy. There are many situations in nanoplasmonics where classical and approximate non-local treatments correctly describe many of the spectral features of the far- and near-field response associated with plasmons; however, new atomic-scale realms in nanophotonics are firmly emerging, as robust technological platforms.

In this chapter, we have focused on quantum-size effects in metallic nanoparticles, and on the optoelectronics of plasmonic gaps. We have shown the differences between tackling the response of noble metals or free metals in the former, and we have established a description of the effect of a tunneling current in screening the gap plasmon, and eventually the formation of a charge transfer plasmon, for the latter. Our description targets the challenge of an accurate quantitative comparison between phenomena identified in cutting-edge experiments and state-of-the-art theoretical descriptions based on TDDFT techniques. The main trends in optical spectroscopy of metal nanoparticles can be unveiled in this way. However, much work remains to be done regarding more sophisticated aspects of optical spectroscopy and optoelectronics. A modification of the tunneling current, and thus of the optical response, in molecular layers has been shown here, but more active ways of controlling the tunneling barriers have called the attention of many researchers in molecular electronics ${ }^{100-102}$ and in electrochemistry. ${ }^{103,104}$ The possibility of controlling the optical response by application of an external bias to a metallic nanogap points out towards new possibilities in optoelectronic devices, and optical routing. ${ }^{105}$ Another aspect of interest is that the tunneling process across a metallic junction is an intrinsic nonlinear process that could be exploited together with other sources of nonlinearity connected with structural and morphological properties of the particles and their environments. All these combined effects can be exploited in novel nanosystems that include plasmonic structures coupled at subnanometric scale. A substantial increase of 
nonlinear signals and the possibility of its control can be expected in this situations. ${ }^{30,84,106,107}$

In addition to the bottom-up approaches to build subnanometric structures, based on wet chemistry methods, scanning probe microscopies also provide a very interesting platform to obtain control of surfaces and molecular species at the atomic-scale. A STM junction where a DC current produces plasmonic excitation and further decays into photons reveals interesting possibilities to modify molecular electroluminescence with unprecedented levels of control. ${ }^{108,109}$ This can lead to the development of new sources of photons for quantum information. Molecular spectroscopy is another field where quantum atomistic effects could be key to understand the subnanometric resolution recently found in experiments which reveals intramolecular features. ${ }^{110}$ Atomistic vertices, edges, and protrusions are able to localize optical fields beyond the standard plasmonic capabilities, ${ }^{39}$ producing a new type of ultraconfined optical cavities which can boost plasmon-exciton coupling ${ }^{111}$ and optomechanical coupling ${ }^{112}$ to values and regimes not explored before.

We have limited the content of this chapter to metallic structures. It is worthwhile to mention that other materials with novel optical properties such as Van der Waals 2D materials, ${ }^{113}$ are also offering the possibility to explore and reveal quantum effects derived from the many-body interaction between the electrons in the system. This is a topic of utmost relevance because of the important technological opportunities; however, we have shown here that standard metallic materials equally hold an incredible potential for optoelectronics and spectroscopy in the optical range of the spectrum.

\section{References}

1. García de Abajo, F. J. (2010). Rev. Mod. Phys. 82, p. 209.

2. Kreibig, U. and Vollmer, M. (1995). Optical Properties of Metal Clusters, (Springer Series in Materials Science) 25.

3. Schuller, J. A., Barnard, E. S., Cai, W., Jun, Y. C., White, J. S. and Brongersma, M. L. (2010). Nat. Mater. 9, p. 193.

4. Alvarez-Puebla, R., Liz-Marzán, L. M. and García de Abajo, F. J. (2010). J. Phys. Chem. Lett. 1, p. 2428. 
5. Stockman, M. I. (2011). Optics Exp. 19, p. 22029.

6. Stewart, M. E., et al. (2008). Chem. Rev. 108, p. 494.

7. Gonçalves, M. R. (2014). J. Phys. D: Appl. Phys. 47, p. 213001.

8. Anker, J. N., Hall, W. P., Lyandres, O., Shah, N. C., Zhao, J. and Van Duyne, R. P. (2008). Nat. Mater. 7, p. 442.

9. Kinkhabwala, A., Yu, Z., Fan, S., Avlasevich, Y., Müllen, K. and Moerner, W. E. (2009). Nat. Photon. 3, p. 654.

10. Baffou, G. and Quidant, R. (2014). Chem. Soc. Rev. 43, p. 3898.

11. Kauranen, M. and Zayats, A. V. (2012). Nat. Photon. 6, p. 737.

12. Liu, N., Hentschel, M., Weiss, T., Alivisatos, A. P. and Giessen, H. (2011). Science 332, p. 1407.

13. Knight, M. W., King, N. S., Liu, L., Everitt, H. O., Nordlander, P. and Halas, N. J. (2014). ACS Nano 8, p. 834.

14. Halas, N. J., Lal, S., Chang, W.-S., Link, S. and Nordlander, P. (2011). Chem. Rev. 111, p. 3913.

15. Savage, K. J., Hawkeye, M. M., Esteban, R., Borisov, A. G., Aizpurua, J. and Baumberg, J. J. (2012). Nature 491, p. 574.

16. Scholl, J. A., García-Etxarri, A., Koh, A. L. P. and Dionne, J. A. (2013). Nano Lett. 13, p. 564.

17. Tan, S. F., Wu, L., Yang, J. K. W., Bai, P., Bosman, M. and Nijhuis, C. A. (2014). Science 343, p. 1496.

18. Hajisalem, G., Nezami, M. S. and Gordon, R. (2014). Nano Lett. 14, p. 6651.

19. Zhu, W. and Crozier, K. B. (2014). Nat. Commun. 5, p. 5228.

20. Apell, P. and Ljungbert, Å. (1982). Solid State Commun 44, p. 1367.

21. Apell, P. and Penn, D. R. (1983). Phys. Rev. Lett. 50, p. 1316.

22. Yannouleas, C. and Broglia, R. A. (1992). Annal. Phys. 217, p. 105.

23. Monreal, C. R., Antosiewicz, T. J. and Apell, S. P. (2013). New J. Phys. 15, p. 083044.

24. Tucker, J. R. and Feldman, M. J. (1985). Rev. Mod. Phys. 57, p. 1055.

25. Platero, G. and Aguado, R. (2004). Phys. Reports 395, p. 1.

26. Ciraci, C., Pendry, J. B. and Smith, D. R. (2013). Chem. Phys. Chem. 14, p. 1109 .

27. Toscano, G. et al. (2015). Nat. Comm. 6, p. 7132.

28. Zuloaga, J., Prodan, E. and Nordlander, P. (2009). Nano Lett. 9, p. 887.

29. Zuloaga, J., Prodan, E. and Nordlander, P. (2010). ACS Nano 4, p. 5269.

30. Marinica, D. C., Kazansky, A. K., Nordlander, P., Aizpurua, J. and Borisov, A. G. (2012). Nano Lett. 12, p. 1333.

31. Esteban, R., Borisov, A. G., Nordlander, P. and Aizpurua, J. (2012). Nat. Commun. 3, p. 825 .

32. Stella, L., Zhang, P., García-Vidal, F. J., Rubio, A. and García-González, P. (2013). J. Phys. Chem. C 117, p. 8941.

33. Teperik, T. V., Nordlander, P., Aizpurua, J. and Borisov, A. G. (2013). Phys. Rev. Lett. 110, p. 263901. 
34. Teperik, T. V., Nordlander, P., Aizpurua, J. and Borisov, A. G. (2013). Opt. Express 21, p. 27306.

35. Andersen, K., Jensen, K. L., Mortensen, N. A. and Thygesen, K. S. (2013). Phys. Rev. B 87, p. 235433.

36. Kulkarni, V., Prodan, E. and Nordlander, P. (2013). Nano Lett. 13, p. 5873.

37. Zhang, P., Feist, J., Rubio, A., García-González, P. and García-Vidal, F. J. (2014). Phys. Rev. B 90, p. 161407(R).

38. Varas, A., García-González, P., García-Vidal, F. J. and Rubio, A. (2015). J. Phys. Chem. Lett. 6, p. 1891.

39. Barbry, M., Koval, P., Marchesin, F., Esteban, R., Borisov, A. G., Aizpurua, J. and Sánchez-Portal, D. (2015). Nano Lett. 15, p. 3410.

40. Feibelman, P. J. (1982). Prog. Surf. Sci. 12, p. 287.

41. Liebsch, A. (1993). Phys. Rev. B 8, p. 11317.

42. Liebsch, A. (1987). Phys. Rev. B 36, p. 7378.

43. Haberland, H. (2013). Nature 494, p. E1.

44. Meier, M. and Wokaun, A. (1983). Opt. Lett. 11, p. 581.

45. Beck, D. E. (1987). Phys. Rev. B 35, p. 7325.

46. Yannouleas, C., Vigezzi, E. and Broglia, R. A. (1993). Phys. Rev. B 47, p. 9849 .

47. Parks, J. H. and McDonald, S. A. (1989). Phys. Rev. Lett. 69, p. 2301.

48. Borggreen, J. et al. (1993). Phys. Rev. B 48, p. 17507.

49. Reiners, T., Ellert, C., Schmidt, M. and Haberland, H. (1995). Phys. Rev. Lett. 74, p. 1558.

50. Charlé, K.-P., Schulze, W. and Winter, B. (1989). Z. Phys. D-Atoms, Molecules and Clusters 12, p. 471.

51. Tiggesbäumker, J., Köller, L., Meiwes-Broer, K.-H. and Liebsch, A. (1993). Phys. Rev. A 48, p. R1749.

52. Charlé, K.-P., König, L., Nepijko, S., Rabin, I. and Schulze, W. (1998). Cryst. Res. Technol. 33, p. 1085.

53. Kasperovich, V. and Kresin, V. V. (1998). Phil. Mag. 78, p. 385.

54. Nilius, N., Ernst, N. and Freund, H.-J. (2000). Phys. Rev. Lett. 84, p. 3994.

55. Borisov, A. G., unpublished.

56. Lermé, J. (2011). J. Phys. Chem. C 115, p. 14098.

57. Serra, L. and Rubio, A. (1997). Phys. Rev. Lett. 78, p. 1428.

58. Jensen, L. L. and Jensen, L. (2009). J. Phys. Chem. C 113, p. 15182.

59. Li, J.-H., Hayashi, M. and Guo, G.-Y. (2013). Phys. Rev. B 88, p. 155437.

60. Iida, K., Noda, M., Ishimura, K. and Nobusada, K. (2014). J. Phys. Chem. A 118, p. 11317 .

61. Kuisma, M. et al. (2015). Phys. Rev. B 91, p. 115431.

62. Romero, I., Aizpurua, J., Bryant, G. W. and García de Abajo, F. J. (2006). Optics Exp. 14, p. 9988.

63. Hövel, H., Fritz, S., Hilger, A., Kreibig, U. and Vollmer, M. (1993). Phys. Rev. B 48, p. 18178. 
64. Klein-Wiele, J.-H., Simon, P. and Rubahn, H.-G. (1998). Phys. Rev. Lett. 80, p. 45.

65. Baida, H. et al. (2009). Nano Lett. 9, p. 3463.

66. Novo, C. et al. (2006). Phys. Chem. Chem. Phys. 8, p. 3540.

67. Wokaun, A., Gordon, J. P. and Liao, P. F. (1982). Phys. Rev. Lett. 48, p. 957.

68. Watanabe, K., Menzel, D., Nilius, N. and Freund, H.-J. (2006). Chem. Rev. 106, p. 4301.

69. Brongersma, M. L., Halas, N. J. and Nordlander, P. (2015). Nat. Nanotechnol. 10, p. 25.

70. Baffou, G. and Quidant, R. (2013). La. Photon. Rev. 7, p. 171.

71. Clavero, C. (2014). Nat. Photon. 8, p. 95.

72. Binnig, G. and Rohrer, H. (1986). IBM J. R. Dev. 30, p. 355.

73. Kravtsov, V., Berweger, S., Atkin, J. M. and Raschke, M. B. (2014). Nano Lett. 14, p. 5270

74. Mertens, J., et al. (2013). Nano Lett. 13, p. 5033.

75. Cha, H., Yoon, J. H. and Yoon, S. (2014). ACS Nano 8, p. 8554.

76. Bochterle, J., Neubrech, F., Nagao, T. and Pucci, A. (2012). ACS Nano 6, p. 10917.

77. Ward, D. R., Hüser, F., Pauly, F., Cuevas, J. C. and Natelson, D. (2010). Nat. Nanotechnol. 5, p. 732.

78. Stolz, A., Berthelo, J., Mennemanteuil, M.-M., Colas des Francs, G., Markey, L., Meunier, V. and Bouhelier, A. (2014). Nano Lett. 14, p. 2330.

79. Ittah, N. and Selzer, Y. (2011). Nano Lett. 11, p. 529

80. Pedersen, M. H. and Büttiker, M. (1998). Phys. Rev. B 58, p. 12993.

81. Grafström, S. (2002). J. Appl. Phys. 91, p. 1717.

82. Dong, T., Ma, X. and Mittra, R. (2012). Appl. Phys. Lett. 101, p. 233111.

83. Haus, J. W., de Ceglia, D., Vincenti, M. A. and Scalora, M. (2014). JOSA $B$ 31, p. 259.

84. Haus, J. W., de Ceglia, D., Vincenti, M. A. and Scalora, M. (2014). JOSA $B$ 31, p. A13.

85. Morton, S. M., Silverstein, D. W. and Jensen, L. (2011). Chem. Rev. 111, p. 3962.

86. Fernández-Domínguez, A. I., Zhang, P., Luo, Y., Maier, S. A., García-Vidal, F. J. and Pendry, J. B. (2012). Phys. Rev. B 86, p. 241110(R).

87. Kottmann, J. and Martin, O. J. F. (2001). Optics Exp. 8, p. 655.

88. Lei, D. Y., Aubry, A., Luo, Y., Maier, S. A. and Pendry, J. B. (2011). ACS Nano 5, p. 597.

89. Pérez-González, O., Zabala, N., Borisov, A. G., Halas, N. J., Nordlander, P., Aizpurua, J. (2010). Nano Lett. 10, p. 3090.

90. Esteban, R., Zugarramurdi, A., Zhang, P., Nordlander, P., Garcia-Vidal, F. J., Borisov, A. G. and Aizpurua, J. (2015). Faraday Discussions 178, p. 151.

91. Simmons, J. G. (1963). J. Appl. Phys. 34, p. 1793.

92. Kulkarni, V. and Manjavacas, A. (2015). ACS Photonics 2, p. 987.

93. Zapata, M., Camacho Beltrán, Á. S., Borisov, A. G. and Aizpurua, J. (2015). Opt. Exp. 23, p. 8134. 
94. Hohenester, U. and Draxl, C. (2016). Phys. Rev. B 94, p. 165418.

95. Galperin, M. and Nitzan, A. (2012). Phys. Chem. Chem. Phys. 14, p. 9421.

96. Song, P., Nordlander, P. and Gao, S. (2011). J. Chem. Phys. 134, p. 074701.

97. Lutz, T. et al. (2013). Nano Lett. 13, p. 2846.

98. Conklin, D. et al. (2013). ACS Nano 7, p. 4479.

99. Benz, F. et al. (2015). Nano Lett. 15, p. 669.

100. Große, C. et al. (2014). Nano Lett. 14, p. 5693.

101. Emboras, A. et al. (2016). Nano Lett. 16, p. 709.

102. Lerch, S. and Reinhard, B. (2016). Adv. Mater. 28, p. 2030.

103. Byers, C. P. et al. (2015). Sci. Adv. 1, p. e1500988.

104. Dong, S., Zhang, K., Yu, Z. and Fan, J. A. (2016). ACS Nano 10, p. 6716.

105. Marinica, D. C. et al. (2015). Sci. Adv. 1, p. e1501095.

106. Danckwerts, M., Novotny, L. (2007). Phys. Rev. Lett. 98, p. 026104.

107. Cai, W., Vasudev, A. P. and Brongersma, M. L. (2011). Science 333, p. 1720

108. Chong, M. C. et al. (2016). Phys. Rev. Lett. 116, p. 036802.

109. Imada, H., Miwa, K., Imai-Imada, M., Kawahara, S., Kimura, K. and Kim, Y. (2016). Nature 538, p. 364.

110. Zhang, R. et al. (2013). Nature 498, p. 82.

111. Chikkaraddy, R. et al. (2016). Nature 535, p. 127.

112. Benz, F. et al. (2016). Science 354, p. 726.

113. Basov, D. N., Fogler, M. M. and García de Abajo, F. J. (2016). Science 354, p. 1992. 